\title{
AN EQUALITY FOR THE CURVATURE FUNCTION OF A SIMPLE AND CLOSED CURVE ON THE PLANE
}

\author{
BIAO OU
}

Received 22 October 2002

\begin{abstract}
We prove an equality for the curvature function of a simple and closed curve on the plane. This equality leads to another proof of the four-vertex theorem in differential geometry. While examining the regularity assumption on the curve for the equality, we make comments on the relation between the boundary regularity of a Riemann mapping and two important subjects, the Schauder theory and the strong maximum principle, for elliptic partial differential equations of second order. We take a note on the curvature function itself in recognizing people's handwriting by a calculating device, as an afterthought on the equality.
\end{abstract}

2000 Mathematics Subject Classification: 30E20, 35J20, 35J65, 53A04.

1. The equality for the curvature function. Let $C$ be a simple and closed curve on the plane. For the convenience of presentation we assume in the beginning that $C$ is analytic. Let $G(z)$ be a one-to-one conformal mapping from the unit disk centered at the origin to the bounded domain enclosed by $C$. Standard complex analysis tells us that $G(z)$ is analytic and

$$
\left|G^{\prime}(z)\right| \geq \mu>0
$$

for a constant $\mu$ on the closed unit disk (cf. [1] or other textbooks). Let

$$
F(z)=G\left(\frac{z-i}{z+i}\right)
$$

and let

$$
\begin{aligned}
& u(z)=\operatorname{Re}\left(\ln \left(F^{\prime}(z)\right)\right)=\ln \left|F^{\prime}(z)\right|, \\
& v(z)=\operatorname{Im}\left(\ln \left(F^{\prime}(z)\right)\right)=\arg \left(F^{\prime}(z)\right) .
\end{aligned}
$$

Then $F(z)$ maps one-to-one the upper half-plane

$$
R_{+}^{2}=\left\{z=\left(x_{1}, x_{2}\right)=x_{1}+i x_{2} \mid x_{2} \geq 0\right\}
$$

to the bounded domain enclosed by $C$, and $u(z), v(z)$ are harmonic functions on $R_{+}^{2}$ functions. 
On the boundary $x_{2}=0, u(z)$ satisfies

$$
u_{x_{2}}\left(x_{1}, 0\right)=-k\left(x_{1}\right) e^{u\left(x_{1}, 0\right)},
$$

where $k\left(x_{1}\right)$ is the curvature of $C$ at $F\left(x_{1}, 0\right)$. This boundary condition follows from the following calculation of the curvature of $C$ :

$$
\begin{aligned}
k\left(x_{1}\right) & =\frac{\operatorname{Im}\left(\overline{F^{\prime}(z)} F^{\prime \prime}(z)\right)}{\left|F^{\prime}(z)\right|^{3}} \quad\left(z=x_{1}=\left(x_{1}, 0\right)\right) \\
& =\operatorname{Im} \frac{\exp (u(z)-i v(z)) \exp (u(z)+i v(z))\left(u_{x_{1}}+i v_{x_{1}}\right)}{\exp (3 u(z))} \\
& =\operatorname{Im} \frac{1}{\exp (u(z))}\left(u_{x_{1}}+i v_{x_{1}}\right) \\
& =\frac{1}{\exp (u(z))}\left(v_{x_{1}}\right) \\
& =\frac{1}{\exp (u(z))}\left(-u_{x_{2}}\right) .
\end{aligned}
$$

Our object is to prove the following equality.

THEOREM 1.1. With $u\left(x_{1}, x_{2}\right)$ and $k\left(x_{1}\right)$ defined as above,

$$
\int_{-\infty}^{\infty} x_{1} k^{\prime}\left(x_{1}\right) e^{u\left(x_{1}, 0\right)} d x_{1}=0
$$

Proof. We first establish the asymptotic behavior of $u(z), v(z)$, and $\nabla u(z)$ at infinity by showing that as $z \rightarrow \infty$,

$$
\begin{aligned}
u(z) & =-2 \ln |z|+O(1), \\
v(z) & =-2 \arg (z)+\arg \left(G^{\prime}(1)\right)+\frac{\pi}{2}+o(1), \\
\nabla u(z) & =-\frac{2 z}{|z|^{2}}+o\left(\frac{1}{|z|}\right) .
\end{aligned}
$$

For this purpose, note that by (1.2)

$$
F^{\prime}(z)=G^{\prime}\left(\frac{z-i}{z+i}\right) \frac{2 i}{(z+i)^{2}}
$$

and that as $z$ approaches infinity,

$$
G^{\prime}\left(\frac{z-i}{z+i}\right) \rightarrow G^{\prime}(1)
$$


with $G^{\prime}(1)$ being nonzero. Thus

$$
\begin{aligned}
u(z)= & \ln \left|F^{\prime}(z)\right|=\ln \left|2 G^{\prime}\left(\frac{z-i}{z+i}\right)\right|-2 \ln |z+i| \\
= & -2 \ln |z|-2 \ln \left|1+\frac{i}{z}\right|+\ln \left|2 G^{\prime}\left(\frac{z-i}{z+i}\right)\right| \\
= & -2 \ln |z|+O(1), \\
v(z)= & \arg \left(F^{\prime}(z)\right)=\arg \left(G^{\prime}\left(\frac{z-i}{z+i}\right)\right)+\frac{\pi}{2}-2 \arg (z+i) \\
= & -2 \arg (z)+\arg \left(G^{\prime}(1)\right)+\frac{\pi}{2}-2 \arg \left(1+\frac{i}{z}\right) \\
& +\left(\arg \left(G^{\prime}\left(\frac{z-i}{z+i}\right)\right)-\arg \left(G^{\prime}(1)\right)\right) \\
= & -2 \arg (z)+\arg \left(G^{\prime}(1)\right)+\frac{\pi}{2}+o(1) .
\end{aligned}
$$

As for $\nabla u(z)$, we have

$$
\begin{aligned}
\frac{\partial u}{\partial x_{1}}-i \frac{\partial u}{\partial x_{2}} & =\frac{\partial u}{\partial x_{1}}+i \frac{\partial v}{\partial x_{1}}=\left(\ln F^{\prime}(z)\right)^{\prime}=\frac{1}{F^{\prime}(z)} F^{\prime \prime}(z) \\
& =\frac{1}{F^{\prime}(z)}\left(G^{\prime}\left(\frac{z-i}{z+i}\right) \frac{-4 i}{(z+i)^{3}}+G^{\prime \prime}\left(\frac{z-i}{z+i}\right)\left(\frac{2 i}{(z+i)^{2}}\right)^{2}\right) \\
& =\frac{-2}{z+i}+\frac{G^{\prime \prime}((z-i) /(z+i))}{G^{\prime}((z-i) /(z+i))} \frac{2 i}{(z+i)^{2}} \\
& =-\frac{2}{z}+\frac{2 i}{z(z+i)}+\frac{G^{\prime \prime}((z-i) /(z+i))}{G^{\prime}((z-i) /(z+i))} \frac{2 i}{(z+i)^{2}} \\
& =-\frac{2}{z}+o\left(\frac{1}{|z|}\right)=-\frac{2 \bar{z}}{|z|^{2}}+o\left(\frac{1}{|z|}\right)
\end{aligned}
$$

Thus we have completed the proof of (1.8).

Next, we recall that $u(z)$ being a harmonic function implies

$$
\frac{\partial}{\partial x_{j}}\left(|\nabla u|^{2} \delta_{i j}-2 \frac{\partial u}{\partial x_{i}} \frac{\partial u}{\partial x_{j}}\right)=0 \text { for each } i=1,2
$$

Here and later the convention of summing over a repeated index is assumed. Even though it is straightforward to verify (1.13), this equation comes out of a well-known argument of calculus of variations. We describe the argument briefly. Let $\phi_{1}$ and $\phi_{2}$ be two functions that are smooth and have a bounded support on $R_{+}^{2}$ and let $\phi=\left(\phi_{1}, \phi_{2}\right)$. For $u_{\epsilon}(z)=u(z+\epsilon \phi(z))$, consider the integral $\int\left|\nabla u_{\epsilon}\right|^{2} d x_{1} d x_{2}$ on a bounded domain that contains the support of $\phi$. 
The derivative of this integral with respect to $\epsilon$ at $\epsilon=0$ equals

$$
\begin{aligned}
\int 2 \nabla u \cdot \nabla\left(\frac{\partial u}{\partial x_{i}} \phi_{i}\right) d x_{1} d x_{2} \\
=\int \frac{\partial}{\partial x_{j}}\left(|\nabla u|^{2} \delta_{i j}-2 \frac{\partial u}{\partial x_{i}} \frac{\partial u}{\partial x_{j}}\right) \phi_{i} d x_{1} d x_{2},
\end{aligned}
$$

after a few elementary manipulations. On the other hand, it equals zero because it is the first variation of the integral involving a harmonic function. Equation (1.13) then follows.

The essential step to equality (1.7) is an integration involving (1.13). Let $B_{R}^{+}$be the upper-half disk centered at the origin with radius $R$. By (1.13) and Green's theorem,

$$
\begin{aligned}
0= & \int_{B_{R}^{+}} x_{i} \frac{\partial}{\partial x_{j}}\left(|\nabla u|^{2} \delta_{i j}-2 \frac{\partial u}{\partial x_{i}} \frac{\partial u}{\partial x_{j}}\right) d x_{1} d x_{2} \\
= & \oint_{\partial B_{R}^{+}} x_{i}\left(|\nabla u|^{2} \delta_{i j}-2 \frac{\partial u}{\partial x_{i}} \frac{\partial u}{\partial x_{j}}\right) v_{j} d l \\
& -\int_{B_{R}^{+}} \delta_{i j}\left(|\nabla u|^{2} \delta_{i j}-2 \frac{\partial u}{\partial x_{i}} \frac{\partial u}{\partial x_{j}}\right) d x_{1} d x_{2} \\
= & \oint_{\partial B_{R}^{+}} x_{i}\left(|\nabla u|^{2} \delta_{i j}-2 \frac{\partial u}{\partial x_{i}} \frac{\partial u}{\partial x_{j}}\right) v_{j} d l .
\end{aligned}
$$

Above $v=\left(v_{1}, v_{2}\right)$ is the outward unit normal vector to $\partial B_{R}^{+}, d l$ is the line integral element, and the line integral is in the counterclockwise direction. Let $I_{1}$ be the line integral on the line segment $\left\{\left(x_{1}, 0\right) \mid-R \leq x_{1} \leq R\right\}$ and let $I_{2}$ be the line integral on the upper-half circle $\{(R \cos \theta, R \sin \theta) \mid 0 \leq \theta \leq \pi\}$. For $I_{1}$, we have $x=\left(x_{1}, 0\right)$ and $v=(0,-1)$; hence

$$
\begin{aligned}
I_{1}= & \int_{-R}^{R} x_{1}\left(|\nabla u|^{2} \delta_{12}-2 \frac{\partial u}{\partial x_{1}} \frac{\partial u}{\partial x_{2}}\right)(-1) d x_{1} \\
= & \int_{-R}^{R} x_{1} 2 \frac{\partial u}{\partial x_{1}}\left(-k\left(x_{1}\right) e^{u\left(x_{1}, 0\right)}\right) d x_{1} \quad(\text { by }(1.5)) \\
= & -\left.2 x_{1} k\left(x_{1}\right) e^{u\left(x_{1}, 0\right)}\right|_{-R} ^{R}+\int_{-R}^{R}\left(2 k\left(x_{1}\right)+2 x_{1} k^{\prime}\left(x_{1}\right)\right) e^{u\left(x_{1}, 0\right)} d x_{1} \\
= & -\left.2 x_{1} k\left(x_{1}\right) e^{u\left(x_{1}, 0\right)}\right|_{-R} ^{R}-\int_{-R}^{R} 2 \frac{\partial u}{\partial x_{2}}\left(x_{1}, 0\right) d x_{1}+\int_{-R}^{R} 2 x_{1} k^{\prime}\left(x_{1}\right) e^{u\left(x_{1}, 0\right)} d x_{1} \\
= & \left(-2 R k(R) e^{u(R, 0)}-2 R k(-R) e^{u(-R, 0)}\right)+2(v(R, 0)-v(-R, 0)) \\
& +\int_{-R}^{R} 2 x_{1} k^{\prime}\left(x_{1}\right) e^{u\left(x_{1}, 0\right)} d x_{1} .
\end{aligned}
$$


For $I_{2}$, we have $x=(R \cos \theta, R \sin \theta)$ and $v=x / R$; hence

$$
I_{2}=\int_{0}^{\pi}\left(|\nabla u|^{2} R^{2}-2 \frac{\partial u}{\partial x_{i}} x_{i} \frac{\partial u}{\partial x_{j}} x_{j}\right) d \theta .
$$

As $R$ approaches infinity, because of (1.8) and that $k(R)$ and $k(-R)$ are bounded, we conclude that

$$
\begin{aligned}
& \left(-2 R k(R) e^{u(R, 0)}-2 R k(-R) e^{u(-R, 0)}\right) \\
& \quad=-2 R k(R) \frac{1}{R^{2}} e^{O(1)}-2 R k(-R) \frac{1}{R^{2}} e^{O(1)} \longrightarrow 0, \\
& 2(v(R, 0)-v(-R, 0)) \\
& \quad=2\left(\left(\arg \left(G^{\prime}(1)\right)+\frac{\pi}{2}+o(1)\right)-\left(-2 \pi+\arg \left(G^{\prime}(1)\right)+\frac{\pi}{2}+o(1)\right)\right) \longrightarrow 4 \pi,
\end{aligned}
$$

and consequently

$$
I_{1} \longrightarrow 4 \pi+\int_{-\infty}^{\infty} 2 x_{1} k^{\prime}\left(x_{1}\right) e^{u\left(x_{1}, 0\right)} d x_{1}
$$

Similarly, as $R$ approaches infinity,

$$
\begin{aligned}
I_{2}= & \int_{0}^{\pi}\left(\frac{\partial u}{\partial x_{i}} \frac{\partial u}{\partial x_{i}} R^{2}-2 \frac{\partial u}{\partial x_{i}} x_{i} \frac{\partial u}{\partial x_{j}} x_{j}\right) d \theta \\
= & \int_{0}^{\pi}\left(\left(-\frac{2 x_{i}}{R^{2}}+o\left(\frac{1}{R}\right)\right)\left(-\frac{2 x_{i}}{R^{2}}+o\left(\frac{1}{R}\right)\right) R^{2}\right. \\
& \left.\quad-2\left(-\frac{2 x_{i}}{R^{2}}+o\left(\frac{1}{R}\right)\right) x_{i}\left(-\frac{2 x_{j}}{R^{2}}+o\left(\frac{1}{R}\right)\right) x_{j}\right) d \theta \\
= & \int_{0}^{\pi}\left(\left(\frac{4}{R^{2}}+o\left(\frac{1}{R^{2}}\right)\right) R^{2}-2(-2+o(1))(-2+o(1))\right) d \theta \\
= & \int_{0}^{\pi}(-4+o(1)) d \theta \rightarrow-4 \pi .
\end{aligned}
$$

Adding the limits of $I_{1}$ and $I_{2}$, we obtain equality (1.7).

2. Remarks on the equality. We make several remarks on the equality.

First, the integral in (1.7) is a proper integral. To see this, let

$$
s=\int_{-\infty}^{x_{1}}\left|F^{\prime}\left(x_{1}, 0\right)\right| d x_{1}=\int_{-\infty}^{x_{1}} e^{u\left(x_{1}, 0\right)} d x_{1}
$$

be a length parameter of $C$. Then

$$
k^{\prime}\left(x_{1}\right)=\frac{d k}{d s} e^{u\left(x_{1}, 0\right)}
$$


Since $d k / d s$ is bounded,

$$
x_{1} k^{\prime}\left(x_{1}\right) e^{u\left(x_{1}, 0\right)}=x_{1} \frac{d k}{d s} e^{2 u\left(x_{1}, 0\right)}
$$

and is, by (1.8), bounded by a constant multiple of $1 /\left(1+\left|x_{1}\right|\right)^{3}$. That is, the integral in (1.7) converges absolutely.

Next, we see how equality (1.7) leads to another proof of the four-vertex theorem in differential geometry. Since we may choose $G(z)$ and $F(z)$ such that $k(0)$ equals the maximum and $k( \pm \infty)$ equal the minimum of the curvature function of $C$, equality (1.7) implies that it is impossible that $x_{1} k^{\prime}\left(x_{1}\right) \leq 0$ for all $x_{1}$. That is, it is impossible that $k\left(x_{1}\right)$ is an increasing function on $-\infty<x_{1}<0$ and a decreasing function on $0<x_{1}<\infty$. Thus $k\left(x_{1}\right)$ has at least two more local maximum or minimum points in addition to 0 and $\pm \infty$. It follows that $C$ has at least four critical points, or vertices. We refer to the papers of Osserman [7] and Tabachnikov [10] for proofs of the four-vertex theorem in differential geometry as well as references to other relevant works.

At this point, we examine the regularity assumption on $C$. Since equality (1.7) involves the first derivative of the curvature function of $C$, the natural assumption is that $C$ is $C^{3}$. We will see that this is also sufficient for (1.7). With (2.3) in mind, we recognize that it is sufficient for all our computations that $G(z)$ be $C^{2, \alpha}$ for some $\alpha$ satisfying $0<\alpha<1$ and (1.1) be satisfied on the closed unit disk. Here we need to mention that although (1.13) involves the third derivative of $G(z)$, we have only used (1.13) in its integral form. Thus by integrating on a half disk slightly above the real line and then taking a limit, we may carry out all the same computations.

Now since $C$ is assumed to be $C^{3}$, it is $C^{2, \alpha}$ for any constant $\alpha$ satisfying $0<\alpha<1$. We need the case $m=2$ of the following theorem.

THEOREM 2.1. If $C$ is $C^{m, \alpha}$ smooth, where $m$ is a positive integer and $0<$ $\alpha<1$, then $G(z)$ is $C^{m, \alpha}$ and $\left|G^{\prime}(z)\right|>0$ on the closed unit disk.

Theorem 2.1 is called Kellogg's theorem in complex analysis. In [11] there is a proof of Theorem 2.1 for the most difficult case $m=1$. It is also known to specialists that Kellogg's theorem can be proved by using the Schauder theory and the strong maximum principle for linear elliptic partial differential equations of second order. However, it is not easy to find a handy reference. My colleague Professor N. V. Rao (Rao Nagisetty) supplied the following proof.

Consider $\ln \left|G^{-1}(z)\right|$, which is a Green's function on the domain enclosed by $C$ with a singularity at $G(0)$. In case $m \geq 2$, applying the standard Schauder theory and the strong maximum principle, as presented in [6], we conclude that $G^{-1}(z)$ is $C^{m, \alpha}$ smooth and the gradient of $G^{-1}(z)$ does not vanish on the closed domain enclosed by $C$. Then the same holds for $G(z)$ on the unit disk by the inverse function theorem. In case $m=1$, the proof goes the same 
way because both the Schauder theory and the strong maximum principle are still valid. Nevertheless, one has to look harder in the literature for a proof in this case. Gilbarg and Trudinger discussed these subjects in the notes of their book [6], and they particularly mentioned works of Finn-Gilbarg and Widman. It seems to be true that it was the study of the boundary regularity of a Riemann mapping that led to the development of the Schauder theory and the strong maximum principle. We refer to the books of Chen and Wu [3] and Giaquinta [5] for different ways to treat the Schauder theory. In addition, there are a lot of works on the Schauder theory for more general elliptic PDEs and on the boundary regularity for disk-type minimal surfaces bounded by a simple and closed space curve. In a series of papers, Professor Friedman extended the Schauder theory to parabolic PDEs, and his work was summarized in [4].

From these discussions, we see that the assumption of $C$ being $C^{3}$ is natural and sufficient for equality (1.7). Consequently, our proof works for the fourvertex theorem for a simple and closed curve that is $C^{3}$ smooth.

With less regularity assumption on the curve, it would be interesting to see what would come out to replace equality (1.7). For example, when a sequence of simple and closed curves that are at least $C^{3}$ smooth converges to a polygon, it remains a problem that what the corresponding equalities (1.7) would converge to.

The higher-dimensional counterpart of equality (1.7) should be the KazdanWarner equality. As a matter of fact, our equality (1.7) is inspired by similar equalities in different contexts. We refer the reader to [2, 9, 12], among many contributions.

In a relevant work [8], the author proved a uniqueness theorem for a harmonic function $u\left(x_{1}, x_{2}\right)$ on the upper half-plane satisfying the boundary condition $u_{x_{2}}\left(x_{1}, 0\right)=-e^{u\left(x_{1}, 0\right)}$ and the constraint $\int_{R_{2}^{+}} e^{2 u} d x_{1} d x_{2}<\infty$. We also refer the reader to a recent paper of Zhang [13].

3. A note on the curvature function itself in the recognition of people's handwriting. The author does not know any other use of equality (1.7). However, while pondering on equality (1.7), I came to think about the problem of recognizing people's handwriting by a calculating device, with the help of the curvature function of a curve.

Let $\left\{(x(t), y(t)) \mid t_{1} \leq t \leq t_{2}\right\}$ be a parametric curve representing a stroke of our handwriting. After discarding what we meant to be a dot or breaking what we wrote into a few segments, we can assume that the curve is smooth. We rescale the curve so that the length is one, and then we calculate the curvature function $k(s), 0 \leq s \leq 1$, where $s$ is the length parameter. The very first theorem of differential geometry tells us that two curves are similar if and only if their curvature functions are identical. Also, it would not be hard to show that two curves resemble each other if their curvature functions are close, a mere corollary of the stability for a linear ordinary differential equation. Thus with 
the help of the curvature function we can make a calculating device to identify what we write! That is, we could build a calculating peripheral that would record what we write as parametric curves and the accompanying software would then recognize what we write. Such a device would help mathematicians to put in writing more easily mathematical symbols and characters that are not convenient to be put into an electronic file on using a keyboard or a mouse.

\section{REFERENCES}

[1] C. Carathéodory, Conformal Representation, Dover Publications, New York, 1998.

[2] W. Chen and C. Li, A note on the Kazdan-Warner type conditions, J. Differential Geom. 41 (1995), no. 2, 259-268.

[3] Y.-Z. Chen and L.-C. Wu, Second Order Elliptic Equations and Elliptic Systems, Translations of Mathematical Monographs, vol. 174, American Mathematical Society, Rhode Island, 1998, translated from the 1991 Chinese original by Bei Hu.

[4] A. Friedman, Partial Differential Equations of Parabolic Type, Prentice-Hall, New Jersey, 1964.

[5] M. Giaquinta, Multiple Integrals in the Calculus of Variations and Nonlinear Elliptic Systems, Annals of Mathematics Studies, vol. 105, Princeton University Press, New Jersey, 1983.

[6] D. Gilbarg and N. S. Trudinger, Elliptic Partial Differential Equations of Second Order, 2nd ed., Springer, New York, 1998.

[7] R. Osserman, The four-or-more vertex theorem, Amer. Math. Monthly 92 (1985), no. 5, 332-337.

[8] B. Ou, A uniqueness theorem for harmonic functions on the upper-half plane, Conform. Geom. Dyn. 4 (2000), 120-125.

[9] S. Pokhozhaev, Eigenfunctions of the equation $\Delta u+\lambda f(u)=0$, Dokl. Akad. Nauk SSSR 165 (1965), 36-39, translation in Soviet Math. Dokl. 6 (1965), 14081411.

[10] S. Tabachnikov, The four-vertex theorem revisited-two variations on the old theme, Amer. Math. Monthly 102 (1995), no. 10, 912-916.

[11] M. Tsuji, Potential Theory in Modern Function Theory, Chelsea, New York, 1975.

[12] H. C. Wente, The differential equation $\Delta x=2 H\left(x_{u} \wedge x_{v}\right)$ with vanishing boundary values, Proc. Amer. Math. Soc. 50 (1975), 131-137.

[13] L. Zhang, Classification of conformal metrics on $\mathbf{R}_{+}^{2}$ with constant Gauss curvature and geodesic curvature on the boundary under various integral finiteness assumptions, Calc. Var. Partial Differential Equations 16 (2003), no. 4, 405430.

Biao Ou: Department of Mathematics, University of Toledo, Toledo, OH 43606, USA

E-mail address: bou@math. utoledo. edu 


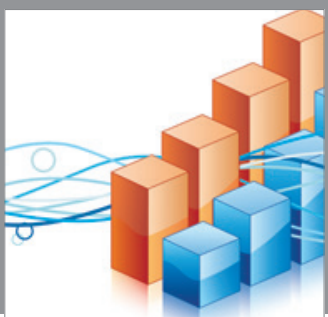

Advances in

Operations Research

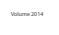

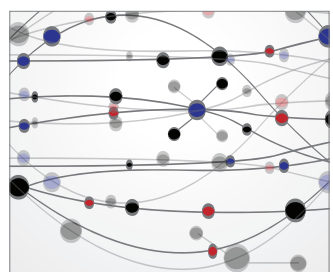

\section{The Scientific} World Journal
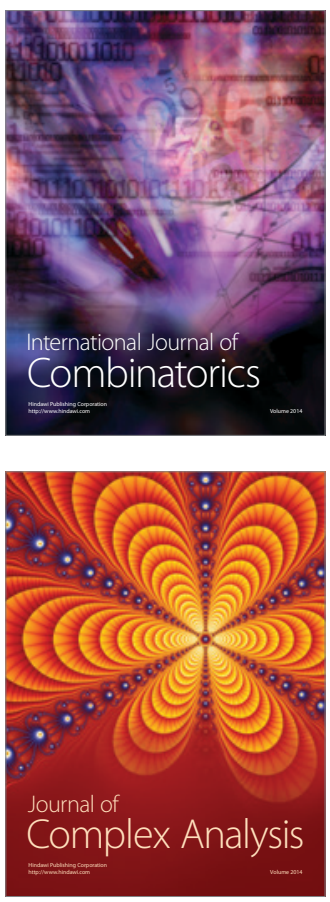

International Journal of

Mathematics and

Mathematical

Sciences
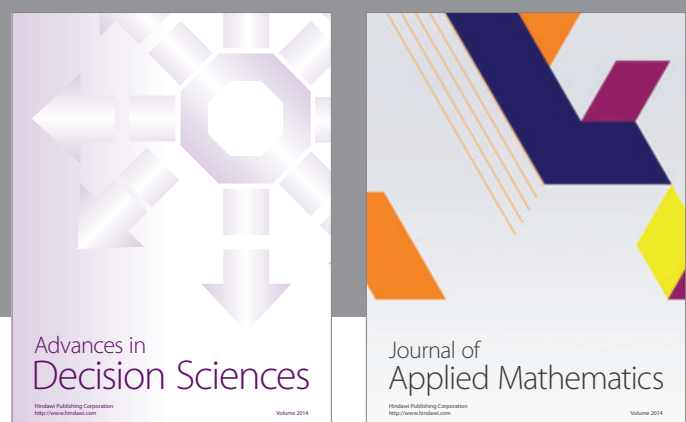

Journal of

Applied Mathematics
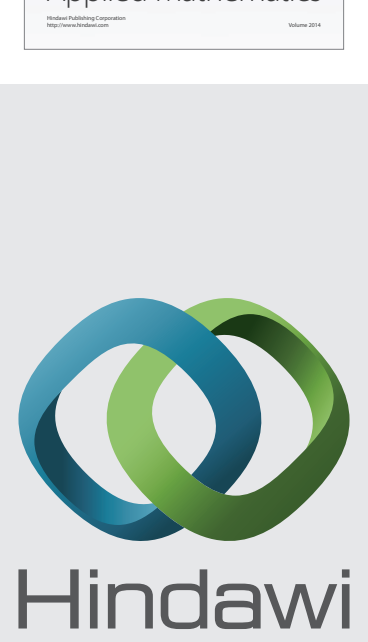

Submit your manuscripts at http://www.hindawi.com
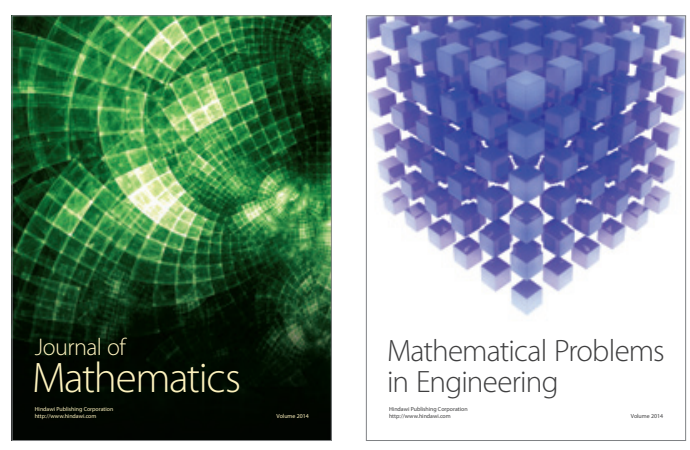

Mathematical Problems in Engineering
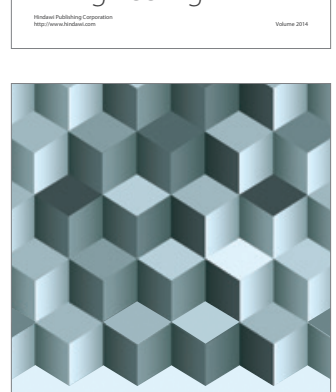

Journal of

Function Spaces
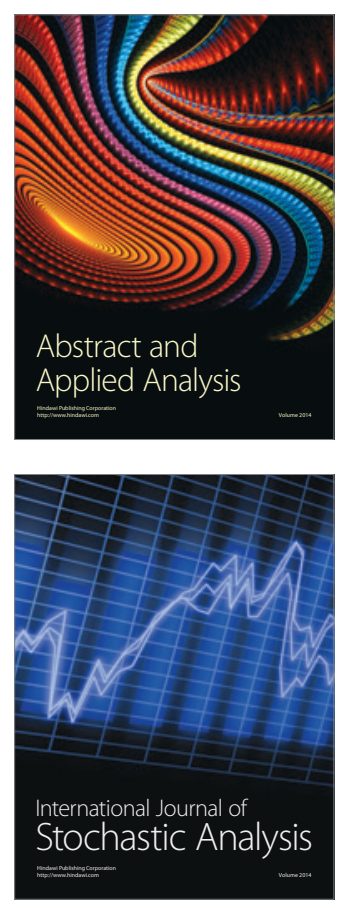

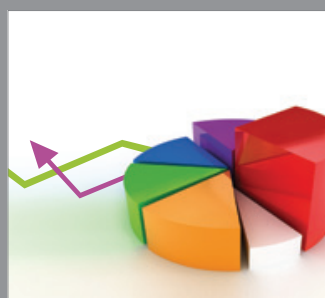

ournal of

Probability and Statistics

Promensencen
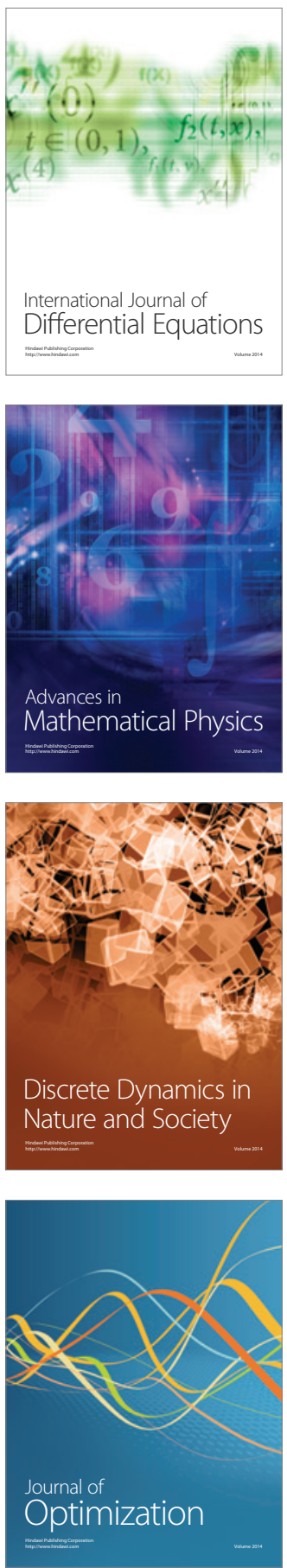